\title{
Performance Analysis of AF and DF for Cooperative Communication using QPSK Modulation over Rayleigh Fading Channel
}

\author{
Bharat Singh Chouhan \\ PG Student \\ Vikrant Institute of Technology \& Management, \\ Indore \\ RGPV University, Bhopal, Madhya Pradesh
}

\author{
Vijay Bisen \\ Asst. Prof. \\ Vikrant Institute of Technology \& Management, \\ Indore \\ RGPV University, Bhopal, Madhya Pradesh
}

\begin{abstract}
The cooperative communication becomes an important research topic in the field of wireless communication technique to improve the speed and reliability of communication over long distance and curvbed surface. The distance is increasing among transmit signal and receiver side signal, the transmitter RF power requirement goes up to maintain the required signal to noise ratio. The main aim is to minimize the bit error rate for Cooperative Communication technique with hierarchical modulation over Rayleigh fading channel. The performance of error rate can be analyzed by every of the transmission bits. The proposed to choose the optimal distance parameters for minimizing the Bit error ratio of the refinement Bit error ratio while guaranteeing the BER requirement of the base bits. The $\mathrm{AF}$ and $\mathrm{DF}$ protocols has been used during simulation. The result of the outage probability with SNR has been shown in result section to identify the effect.
\end{abstract}

\section{Keywords}

Cooperative Communication, AF and DF, QPSK, Rayleigh channel, Outage Probability, BER, SNR, etc

\section{INTRODUCTION}

The increased demand for wireless communications applications has significant development of wireless networks, in the particular various Cellular system that are hungry for voice and data resources and more recently The Ad-hoc networks. The radio equipment and wireless services are evolution to become more efficient and cheaper for its users thanks to the work and studies researchers and experts who have a better understanding of the Transmission and generally multi-user communications technology. And the wired communications system, we begin to understand the fundamental limits the performance of wireless networks and the practical methods of approaching it.

The cooperative communication technique uses single antenna mobiles to reap some of the benefits of multiple input and multiple output (MIMO). The basic idea is that single antenna mobiles in a multi-user scenario can "share" their antennas in a manner that creates a virtual multiple input and multiple output. It is a promising technique to overcome fading and interference in wireless environment. By combining signals through several paths from several users in receiver node, both spatial diversity and user diversity are fully exploited. The capacity of the three-node network consisting of a source, a destination, and a relay has been analyzed. The assumed that all nodes operate in the same band, so the scheme can be decomposed into a broadcast channel from the viewpoint of the source and a multiple access channel from the viewpoint of the destination. Many ideas that appeared later in the cooperation literature were first exposited in it. Although transmit diversity is clearly advantageous on a cellular base station (BS), but it may not be practical for certain other scenarios like wireless sensor networks (WSN), mobile adhoc networks and other. Specifically, due to size, cost, power or hardware limitations, a wireless agent may not be able to support multiple transmit and receive antennas.

There are many protocols and architectures of RF energy transfer applied in wireless powered network has introduced in literature. As a first instance, relay nodes are able to collect energy from source node and utilize it to forward the data to the destination node. The solution of prolonging the lifetime by energy harvesting for the amplify-and-forward (AF) relaying network through power constrained relay nodes has investigated [1].

\section{COOPERATIVECOMMUNICATION TRANSMISSION}

The Cooperative communication technique includes the source, destination, and relays in among both of them. The assumed that all terminals are equipped with a single antenna and can't transmit and receive simultaneously for fading channel coefficients are denoted through hsd, hsr, and hrd,

$$
\mathrm{F}(\mathrm{x} ; \mathrm{m}, \Omega)=2 \mathrm{~m}^{2} \mathrm{x}^{2 \mathrm{~m}-1} \exp \left(-\frac{\mathrm{mx}^{2}}{\Omega}\right) / \Gamma(\mathrm{m}) \Omega^{\mathrm{m}}
$$

Consider the dual-hop relaying scenario in Fig. 1. Let the transmission parameters between the source and the relay have subscript 1 and between relay and destination have subscript 2 . Using the generalized system model, the received signals at the relay and destination are

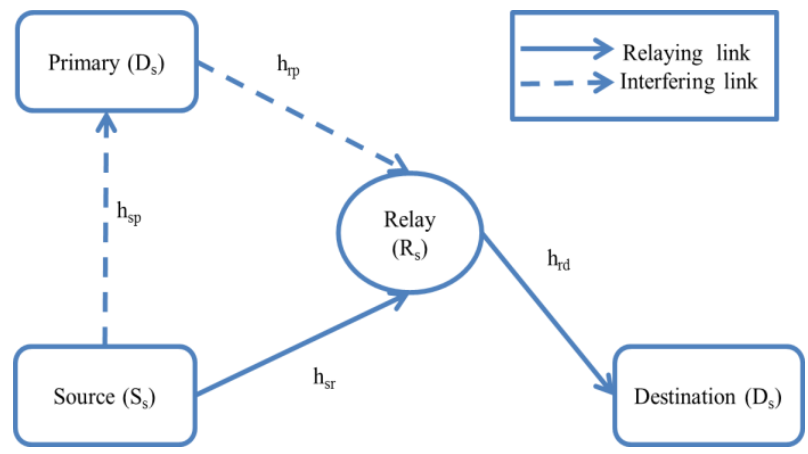

Fig: 1 Dual-hop relay Cooperative communication 
The cooperative transmission protocols used at the relay are amplify and forward (AF), and decode and forward (DF), compress and forward and coded cooperation. The most commonly used strategies are AF and DF.

\section{Amplify and Forward}

In this case the relay forwards the information received from the sender during broadcast phase and it amplifies and retransmit the signal to its destination during the cooperation phase. In doing so, noise also get amplified, which is the major drawback of this method. This method is used when the time delay caused by decoding and encoding is to be minimized.

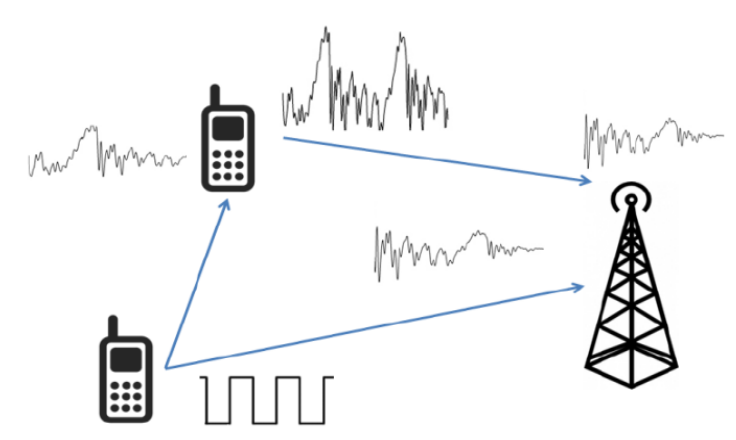

Fig: 2 Amplify and Forward

The relay has to send the signal at the same power level at which it received the signal; hence the relay has to use a gain of

$\beta_{\mathrm{r}}=\frac{\sqrt{\mathrm{P}}}{\sqrt{\mathrm{P}\left|\mathrm{h}_{\mathrm{s}, \mathrm{r}}\right|^{\wedge} 2+\mathrm{N}_{0}}}+\eta_{\mathrm{s}, \mathrm{r}}$

Thus signal received at the destination is

$\mathrm{y}_{\mathrm{r}, \mathrm{d}}=\frac{\sqrt{\mathrm{P}}}{\sqrt{\mathrm{P}\left|\mathrm{h}_{\mathrm{s}, \mathrm{r}}\right|^{\wedge} 2+\mathrm{N}_{0}}}+\mathrm{h}_{\mathrm{r}, \mathrm{d}} \mathrm{y}_{\mathrm{s}, \mathrm{r}}+\eta_{\mathrm{s}, \mathrm{r}}$

$\mathrm{y}_{\mathrm{r}, \mathrm{d}}=\frac{\sqrt{\mathrm{P}}}{\sqrt{\mathrm{P}\left|\mathrm{h}_{\mathrm{s}, \mathrm{r}}\right|^{\wedge} 2+\mathrm{N}_{0}}}+\mathrm{h}_{\mathrm{r}, \mathrm{d}} \sqrt{\mathrm{P}} \mathrm{h}_{\mathrm{s}, \mathrm{r}} \mathrm{x}+\eta_{\mathrm{r}, \mathrm{d}}^{\prime}$

Where equivalent noise is given as:

$\eta_{r, d}^{\prime}=\frac{\sqrt{\mathrm{P}}}{\sqrt{\bar{P}\left|\mathrm{~h}_{\mathrm{s}, \mathrm{r}}\right|^{\wedge} 2+\mathrm{N}_{0}}}+\mathrm{h}_{\mathrm{r}, \mathrm{d}} \eta_{\mathrm{s}, \mathrm{r}}^{\prime}+\eta_{\mathrm{r}, \mathrm{d}}$

\section{Decode and Forward}

In this scheme, the relay decodes the information received from the source during the broadcast phase before forwarding it to its destination. After successfully decoding the received signal, the relay re-encodes the signal and forwards it to the destination [1].So there is no amplified noise in the received signal. The received information at the receiver via relay can be expressed as:

$\mathrm{y}_{\mathrm{r}, \mathrm{d}}=\mathrm{x} \cdot \mathrm{h}_{\mathrm{r}, \mathrm{d}}+\eta_{\mathrm{r}, \mathrm{d}}$

The decode and forward method can be implemented in two ways. One is when the relay can completely decode the message, but this requires a lot of time. If there is an error correcting code in the source message, then the received bit errors can be corrected at the relay station.

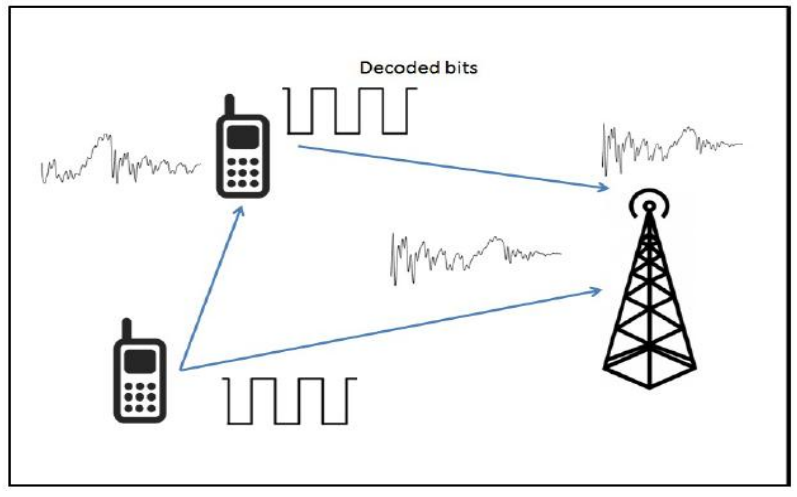

Fig: 3 Decode and Forward

But it is not possible to always completely decode the source message, since this requires a lot of computing time and the additional delay caused might be unacceptable. In such a case, the source message is just decoded and re-encoded symbol by symbol. So no error correcting code can be applied.

\section{SYSTEM MODEL}

The simulation model consist dual hop relaying system in the relaying fading environment. The ideal hardware system has been used initially used for the simulation for the symbols more than $10^{\wedge} 5$. The figure below shows a schematic diagram of the dual hop transmission. The out can be modeled as

$$
y=h(s+\eta)+v
$$

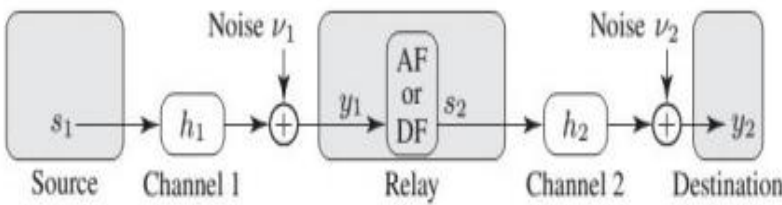

Fig: 4 Dual-hop relaying scenario for ideal hardware case

There are no impurity in the signal introduced by source or by relay channel. There are different type of imperfectness has been observed in practice. This paper deals with the imperfectness at source side and relay side as shown in the figure below,
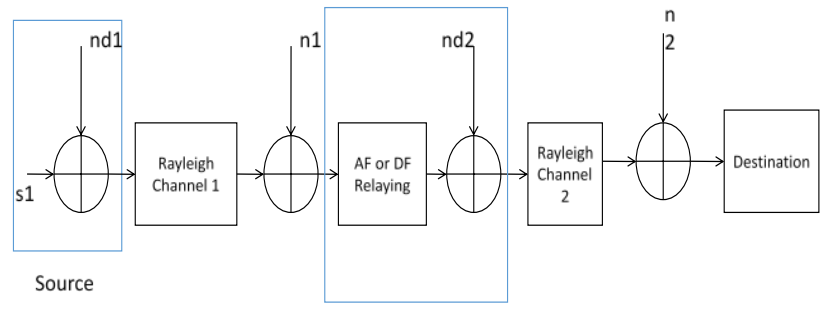

Relay

Fig: 5 Dual-hop relaying scenario for non-ideal hardware case

The nd 1 and nd 2 are the noises added during the transmission. The gain of the AF technique influence due to the noise variance and an addition gain error also introduce in the transmission from source and relay 


\section{RESULTS AND DISCUSSION}

The performance of cooperative communication and AF DF protocol is analyzed when direct link is used for transmission of signals, when single relay is used and when two relays are used and all signals are combined at receiver. MATLAB software platform is use to perform the experiment. Two parameters Bit error ratio and outage probability has been used in the paper. The BER is the ratio of no. of error bit with respect to total transmitted bit. The outage probability is described There are four different case has been considered in this paper and the outage probability is define as below

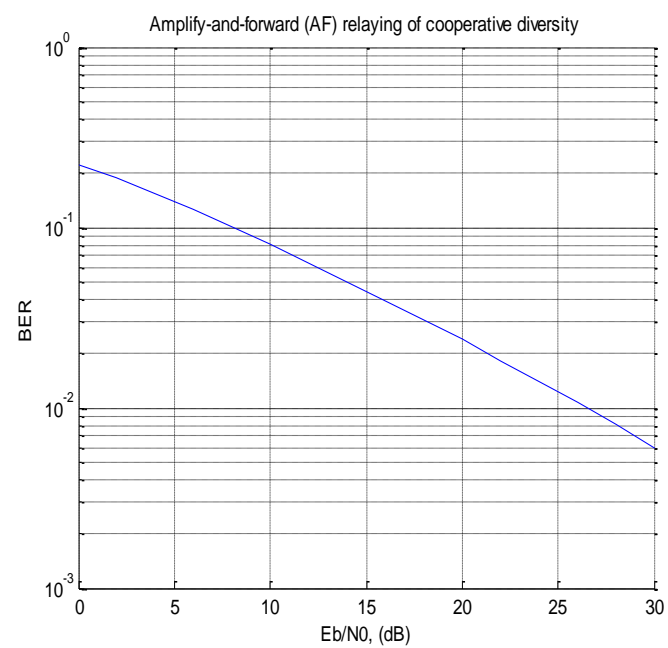

Fig: 5 Performance of SNR Vs BER

Fig. 6 show in modulation technique with cooperative communication, Effect of modulation order on the BER of AF cooperative communication.

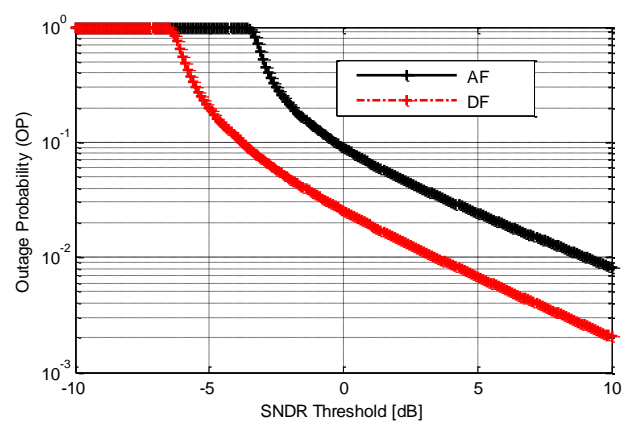

Fig: 6 Performance of outage probability for $\mathrm{AF}$ and DF relaying

\section{CONCLUSION}

In this paper performance of AF cooperative communication technique is investigated. It is observed that when two relays are used instead of single relay, there is a reduction in bit error rate.

- It has been shown that cooperative diversity brings several benefits including better signal quality, reduced transmission power, better coverage and higher capacity.
- Cooperative diversity has some drawbacks and challenges including resource over-utilization, additional delay, implementation complexity, unavailability of cooperating nodes and security threats.

\section{REFERENCES}

[1] Sadek, A.K.; Zhu Han; Liu, K.J.R., "A Distributed Relay-Assignment Algorithm for Cooperative Communications in Wireless Networks," Communications, 2006. ICC '06.IEEE International Conference on, vol.4, no., pp.1592, 1597, June 2006.

[2] Sudharman K. Jayaweera, "Energy Efficient Virtual MIMO-based Cooperative Communications for Wireless Sensor Networks",

[3] Laneman, J.N.; Tse, D.N.C.; Wornell, Gregory W., "Cooperative diversity in wireless networks: Efficient protocols and outage behavior," Information Theory, IEEE Transactions on, vol.50, no.12, Dec. 2004.

[4] Anghel, Paul A., and Mostafa Kaveh. "Exact symbol error probability of cooperative network in a Rayleighfading environment." Wireless Communications, IEEE Transactions on 3.5 (2004): 1416-1421.

[5] Simon, Marvin K., and Mohamed-Slim Alouini. "A unified approach to the performance analysis of digital communication over generalized fading channels." Proceedings of the IEEE 86.9 (1998): 1860-1877.

[6] Yuan, Runping, et al. "Opportunistic cooperation and optimal power allocation for wireless sensor networks." Consumer Electronics, IEEE Transactions on56.3 (2010):1898-1904.

[7] Emil Bjornson,A New Look at Dual-Hop Relaying: Performance Limits with Hardware Impairments , IEEE Transactions On Communications, Vol. 61, No. 11, November 2013

[8] Suraweera, H.A., Garg, H.K. and Nallanathan, A., "Performance analysis of two hop amplify-and-forward systems with interference at the relay." IEEE Communications Letters, 14(8), 2010.

[9] Zhou, S., Liu, H., Zhao, Y. and Hu, L., "Target spatial and frequency scattering diversity property for diversity MIMO radar”. Signal Processing, 91(2), 2011.

[10] Lozano, A. and Jindal, N., "Transmit diversity vs. spatial multiplexing in modern MIMO systems". IEEE Transactions on Wireless Communications, 2010.

[11] Di Renzo, M., Haas, H., Ghrayeb, A., Sugiura, S. and Hanzo, L."Spatial modulation for generalized MIMO: Challenges, opportunities, and implementation". Proceedings of the IEEE, 102(1), 2014.

[12] Krishnaswamy, H. and Hashemi, H., "A 4-channel 4beam 24-to-26GHz spatio-temporal RAKE radar transceiver in $90 \mathrm{~nm}$ CMOS for vehicular radar applications. In Solid-State Circuits". Conference Digest of Technical Papers (ISSCC), IEEE International, 2010. 